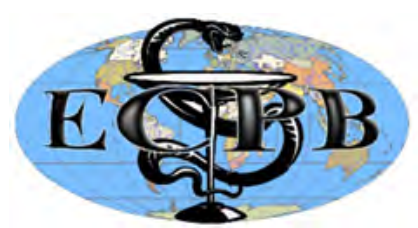

"ЕКСПЕРИМЕНТАЛЬНА ТА КЛІНІЧНА ФІЗІОЛОГІЯ І БІОХІМІЯ" "EXPERIMENTAL AND CLINICAL PHYSIOLOGY AND BIOCHEMISTRY" Науково-практичний журнал/Scientific-practical journal

Наукові статті / Research article ECPB 2018, 1(81): 11-26. https://doi.org/

\title{
Immediate responses of the autonomic nervous system to the balneofactors, their neuro-endocrine- immune accompaniments and predictors
}

\author{
I.L. POPOVYCH \\ Bohomolets' Institute of Physiology of National Academy of Sciences, Kyiv, Ukraine
}

\section{E-maul: i.popovych@biph.kiev.ua}

Introduction. In previous studies of the Truskavets' Scientific School of Balneology, it has been shown that in response to the use of Bioactive Water Naftussya (BAWN) the activity of the autonomic nervous system changes. However, the nature of the reaction is ambiguous and is conditioned by the constellation of the initial parameters of the body $[15,16,24,28]$. The immediate reactions of diuresis, gastric and pancreatic secretion, hemodynamics were also interspersed $[2,6,9,27,28,31]$. In the context of the adaptogenic concept of the mechanism of action BAWN [22], it is also shown that the polyversive reactions of the autonomic nervous system are accompanied by changes in some parameters of the electroencephalogram, immunogram of blood and levels of adaptation hormones in it [14-16, 24, 28]. However, the issue of the specificity of immediate effects of BAWN on the nervous, endocrine and immune systems, that function interconnected as a triple complex $[15,21,22,28]$, is still relevant. The answer to this question can be obtained by comparing the reactions of the autonomic nervous system to BAWN with reactions to ordinary (daily) water - on the one hand, and other natural adaptogens - on the other hand. This is the objective of this study.

Material and Methods. The objects of the observation were 15 volunteers-men (aged $26 \div 60$ years, $\mathrm{M} \pm \mathrm{SD}: 44 \pm 12$ years) without any clinical diagnose but with a moderate dysfunction of neuroendocrine-immune complex (dysadaptation).

At first volunteers filled in a questionnaire with the purpose of estimation of the level of the trait anxiety [29]. Then EEG was recorded for $25 \mathrm{sec}$ by means of the hardware-software complex 'NeuroCom Standard' (producer KhAI Medica, Kharkiv, Ukraine) monopolar in 16 loci (Fp1, Fp2, F3, F4, F7, F8, C3, C4, T3, T4, P3, P4, T5, T6, O1, O2) by 10-20 international system, with the reference electrodes $\mathrm{A}$ and Ref on tassels of the ears. Among the options the average EEG amplitude $(\mu \mathrm{V})$ was considered as well as the modal frequency $(\mathrm{Hz})$, frequency deviation $(\mathrm{Hz})$, index $(\%)$, coefficient of asymmetry $(\%)$, absolute $(\mu \mathrm{V} 2 / \mathrm{Hz})$ and relative (\%) power spectrum density (SPD) of basic rhythms: 8 $(35 \div 13 \mathrm{~Hz})$, a $(13 \div 8 \mathrm{~Hz}), \theta(8 \div 4 \mathrm{~Hz})$ and $\delta(4 \div 0,5 \mathrm{~Hz})$ in all loci, according to the instructions of the device. In addition, the Laterality Index (LI) for SPD of each rhythm was calculated using the formula [18]:

$$
\text { LI, } \%=\Sigma[200 \cdot(\text { Right }- \text { Left }) /(\text { Right }+ \text { Left })] / 8 \text {. }
$$

For each locus the Entropy (h) of normalized SPD was also calculated by using classical CE Shannon's formula [30]:

$$
\begin{gathered}
\mathrm{h}=-\left[\mathrm{SPDa} \cdot \log _{2} \mathrm{SPDa}+\mathrm{SPDB} \cdot \log _{2} \mathrm{SPDB}+\mathrm{SPD} \cdot \cdot \log _{2} \mathrm{SPD} \theta+\right. \\
\left.+\mathrm{SPD} \delta \cdot \log _{2} \mathrm{SPD} \delta\right] / \log _{2} 4
\end{gathered}
$$

(C) Popovych I.L., 2017 
Then the electrocardiogram in II lead was recorded for 7 min to assess the parameters of the heart rate variability (HRV) (the hardware-software complex "CardioLab+HRV" "KhAI-MEDICA", Kharkiv). For further analysis the following parameters HRV were selected: a) Bayevskiy's parameters: heart rate $(\mathrm{HR})$, the moda (Mo), the amplitude of moda (AMo), variational sweep (MxD$\mathrm{Mn})$, Stress Index (BSI=AMo/2 $\left.\bullet \mathrm{Mo}^{\circ} \cdot \mathrm{MxDMn}\right)$ as well as the Activity Regulatory Systems Index (BARSI) [1]; b) Temporal parameters (Time Domain Methods): the standard deviation of all NN intervals (SDNN), the square root of the sum of the squares of differences between adjacent NN intervals (RMSSD), the percent of interval differences of successive NN intervals is greater than $50 \mathrm{~ms}$ (pNN50), triangulary index (TNN); c) Spectral parameters (Frequency Domain Methods): SP of HRV bands: high-frequency (HF, range $0,4 \div 0,15 \mathrm{~Hz}$ ), low-frequency $(\mathrm{LF}$, range $0,15 \div 0,04 \mathrm{~Hz}$ ), very low-frequency (VLF, range $0,04 \div 0,015$

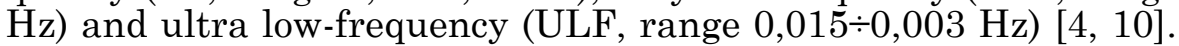

The Leukocytogram counted up in the portion of capillary blood and its Adaptation as well as Strain Indexes by I.L. Popovych were calculated [3, 20]. The phagocytic function of neutrophils can be judged by the activity (percentage of neutrophils, in which found microbes - Phagocytic Index, PhI), intensity (number of microbes absorbed in one phagocyte - Microbial Count, MC) and the completeness (percentage of dead microbes - Killing Index, KI) [5, 8, 22] of phagocytosis museum culture Staphylococcus aureus (ATCC N 25423 F49) obtained from the Truskavets' Laboratory of Hydrogeological Regime-Operational Station spa. Based on these parameters the bactericidal capacity of blood neutrophils (BCCN) was calculated by using the formula [22]:

$$
\begin{aligned}
\operatorname{BCCN}\left(10^{9} \mathrm{Micr} / \mathrm{L}\right)= & \text { Leukocytes }\left(10^{9} / \mathrm{L}\right) \cdot \operatorname{Neutrophils}(\%) \cdot \operatorname{PhI}(\%) \cdot \\
& \cdot \mathrm{MC}(\text { Micr } / \text { Phag }) \cdot \mathrm{KI}(\%)
\end{aligned}
$$

The immune status was evaluated on a set of I and II levels recommended by the WHO. For phenotyping subpopulations of lymphocytes such methods were used as the method of rosette formation and indirect immunofluorescent binding reaction of monoclonal antibodies from the company "Sorbent" $(\mathrm{RF})$ with visualization under the fluorescent microscope. T-cellular immunity was assessed by the following parameters: blood levels of a subpopulation of "active", theophilline resistance and sensitive T-lymphocytes as well as lymphocytes phenotype of $\mathrm{CD}^{+} \mathrm{CD}^{+}\left(\mathrm{T}\right.$-helper) and $\mathrm{CD}^{+} \mathrm{CD}^{+}$(T-cytolytic). In addition $\mathrm{CD} 16^{+}$-lymphocytes (natural killers) were determined. The state of the humoral immunity was evaluated by the content of CD $19^{+}$B-lymphocytes and concentration of immunoglobulins classes $\mathrm{G}, \mathrm{A}, \mathrm{M}$ in serum (the radial immunodiffusion method) and circulating immune complexes (with polyethylene glycol precipitation method) by using standardized methods described in the manual [17].

In plasma of venous blood the content of principal adaptation Hormones was determined such as Cortisol, Testosterone and Triiodothyronine (by the ELISA with the help of the analyzer "Tecan", Oesterreich) and the corresponding sets of reagents from "AlkorBio", $\mathrm{RF}$ as well as $\mathrm{Na}^{+}$and $\mathrm{K}^{+}$(by the method of flaming photometry with the use of "SF-46" PFMU 4.2 for evaluating the Mineralocorticoide activity as $(\mathrm{Na} / \mathrm{K})^{0,5}$ Ratio [24].

After the registration on Basal Level 5 volunteers for some days consumed $200 \mathrm{~mL}$ of Control Waters (distillated, filtered, well), Water Naftussya from the layers of Truskavets' and Skhidnyts'a, while 10 volunteers consumed $5 \mathrm{~mL}$ of Phytocomposition "Balm Cryms'kyi" (it is identified as an adaptogen [19]) soluted in $195 \mathrm{~mL}$ of daily Water. All tests were repeated in an hour and a half.

Normal values have been borrowed from the instructions for devices and sets as well as databases of the Truskavets' Scientific School.

Results were processed by using the software package "Statistica 5.5".

Results and discussion. The cohort characterized by dysadaptation was observed, high frequentlyties of Dysharmonious General Adaptation Reactions 
(53,7\%) and Distress (11,1\%) were documented, Popovych's Leukocytary Adaptation Index $(1,03 \pm 0,08$ points vs range of norm $1,46 \div 1,95)$ has decreased, while Popovych's Leukocytary Strain Index $(0,35 \pm 0,07$ vs 0 in norm) has increased as well as Bayevskiy's Stress Index (5,34 $\pm 0,15$ vs $4,82 \pm 0,03$ ln units in norm) and Bayevskiy's Activity Regulatory Systems Index (4,62 $\pm 0,82$ units vs range of norm $0 \div 3$ ).

As the integral response criterion of the autonomic nervous system, Bayevskiy's Stress Index is changing based on our preliminary data [25] that this HRV parameter correlates with EEG parameters more closely than the LF/ $\mathrm{HF}$ ratio: $\mathrm{R}=0,80$ vs 0,56 . The preliminary analysis confirmed the polyvariant nature of the vegetotropic reactions to the stimulus, namely: Bayevskiy's Stress Index has increased in half of observations, while in 39\% it decreased, and only in $11 \%$ was not significantly changed. Despite the expectations, no differences were found between the effects of all applied stimuli, so they were not considered separately at the next stage of the analysis.

The vagotonic reaction is accompanied by the increase of Variational Sweep (Table 1), SDNN, Triangulary Index and SP LF band HRV (Table 2) in combination with the decrease of Amplitude of Moda (Table 1). On the contrary, the sympathetic response is associated with the decrease of Variational Sweep, Moda, SDNN, RMSSD, pNN50, Triangulary Index and SP of all bands HRV in combination with the increase of Amplitude of Moda, Heart Rate and Bayevskiy's Activity Regulatory Systems Index. The neutral vegetotropic reaction has not changed any of the parameters of the HRV, which is quite understandable.

Table 1

Comparative characteristics of Bayevskiy's parameters of Heart Rate Variability

\begin{tabular}{|c|c|c|c|c|c|}
\hline \multirow{2}{*}{ Variables } & Term & \multicolumn{2}{|c|}{ Immediate vegetotropic reaction (n) } & \multirow{2}{*}{$\begin{array}{c}\text { Norma- } \\
\text { tives } \\
(\mathbf{n = 5 4 )}\end{array}$} \\
\cline { 3 - 6 } & & Vagotonic (21) & Neutral (6) & Sympathotonic (27) & \\
\hline Bayevskiy's & Before & $5,92 \pm 0,30^{*}$ & $5,53 \pm 0,22^{*}$ & $4,86 \pm 0,12$ & $4,82 \pm 0,03$ \\
Stress Index, & After & $5,35 \pm 0,22^{*}$ & $5,57 \pm 0,19^{*}$ & $5,37 \pm 0,11^{*}$ & \\
ln units & Change & $-0,57 \pm 0,14^{\#}$ & $+0,04 \pm 0,04$ & $+0,61 \pm 0,08^{\#}$ & \\
\hline Amplitude of & Before & $66 \pm 5^{*}$ & $55 \pm 6^{*}$ & $43 \pm 3$ & $37,8 \pm 1,3$ \\
Moda, & After & $51 \pm 4^{*}$ & $55 \pm 6^{*}$ & $58 \pm 4^{*}$ & \\
$\%$ & Change & $-15 \pm 3^{\#}$ & $0 \pm 2$ & $+15 \pm 3^{\#}$ & \\
\hline Variational & Before & $160 \pm 21^{*}$ & $154 \pm 19^{*}$ & $210 \pm 12^{*}$ & $252 \pm 8$ \\
Sweep, & After & $185 \pm 22^{*}$ & $147 \pm 17^{*}$ & $170 \pm 118$ & \\
msec & Change & $+25 \pm 9^{\#}$ & $-6 \pm 7$ & $-40 \pm 8^{\#}$ & \\
\hline Moda, & Before & $784 \pm 34^{*}$ & $728 \pm 45^{*}$ & $808 \pm 27$ & $864 \pm 14$ \\
msec & After & $772 \pm 36^{*}$ & $728 \pm 31^{*}$ & $750 \pm 26^{*}$ & \\
& Change & $-12 \pm 18$ & $0 \pm 37$ & $-58 \pm 17^{\#}$ & \\
\hline Heart Rate, & Before & $76,8 \pm 3,5$ & $80,4 \pm 4,1^{*}$ & $74,6 \pm 2,3$ & $69,3 \pm 1,5$ \\
beats/min & After & $77,9 \pm 3,1^{*}$ & $81,7 \pm 2,8^{*}$ & $80,0 \pm 2,6^{*}$ & \\
& Change & $+1,1 \pm 1,5$ & $+1,3 \pm 3,2$ & $+5,4 \pm 1,5^{\#}$ & \\
\hline
\end{tabular}

* Average values that are significant $(p<0,05)$ differ from normal, are indicated.

\# Changes are calculated as direct differences between individual values after and before drinking. Significant changes are marked.

Regarding the concomitant changes in the hormones of adaptation, a significant increase in Testosterone levels has been observed after the vagotonics reaction and a tendency towards the decrease in the sympathetic response, however, with the neutral reaction the changes have been uncertain. Equally uncertain were the changes on levels of Cortisol and Triiodothyronine as well as the Mineralocorticoid activity (Table 3). The Popovych's Leukocytary Strain index was also significantly reduced only in the vagotonic response which reflects the decrease in the deviation from the optimum zone of elements of Leukocytogram, mainly monocytes. 
Table 2

Comparative characteristics of Temporal and Spectral parameters of Heart Rate Variability

\begin{tabular}{|c|c|c|c|c|c|}
\hline \multirow{2}{*}{ Variables } & \multirow{2}{*}{ Term } & \multicolumn{3}{|c|}{ Immediate vegetotropic reaction (n) } & \multirow{2}{*}{$\begin{array}{c}\text { Norma- } \\
\text { tives } \\
(n=54)\end{array}$} \\
\hline & & Vagotonic (21) & Neutral (6) & Sympathotonic (27) & \\
\hline $\begin{array}{l}\text { SDNN, } \\
\text { msec }\end{array}$ & $\begin{array}{l}\text { Before } \\
\text { After } \\
\text { Change }\end{array}$ & $\begin{array}{l}35 \pm 3^{*} \\
41 \pm 5^{*} \\
+6 \pm 2^{\#}\end{array}$ & $\begin{array}{l}34 \pm 5^{*} \\
32 \pm 4^{*} \\
+3 \pm 3\end{array}$ & $\begin{array}{c}47 \pm 3^{*} \\
36 \pm 3^{*} \\
-11 \pm 2 \#\end{array}$ & $57 \pm 2$ \\
\hline $\begin{array}{l}\text { RMSSD, } \\
\text { msec }\end{array}$ & $\begin{array}{l}\text { Before } \\
\text { After } \\
\text { Change }\end{array}$ & $\begin{array}{l}22 \pm 3^{*} \\
23 \pm 3^{*} \\
+1 \pm 1\end{array}$ & $\begin{aligned} 16 & \pm 3^{*} \\
16 & \pm 2^{*} \\
0 & \pm 3\end{aligned}$ & $\begin{array}{l}28 \pm 3 \\
22 \pm 3^{*} \\
-6 \pm 2^{\#}\end{array}$ & $32 \pm 3$ \\
\hline $\begin{array}{c}\text { pNN50, } \\
\%\end{array}$ & $\begin{array}{l}\text { Before } \\
\text { After } \\
\text { Change }\end{array}$ & $\begin{array}{c}5,3 \pm 2,5 \\
5,9 \pm 2,6 \\
+0,6 \pm 0,4\end{array}$ & $\begin{array}{c}1,6 \pm 1,0^{*} \\
1,6 \pm 0,6^{*} \\
0,0 \pm 0,9\end{array}$ & $\begin{array}{c}8,8 \pm 2,7 \\
4,8 \pm 2,1 \\
-4,0 \pm 1,5^{\#}\end{array}$ & $10,6 \pm 2,2$ \\
\hline $\begin{array}{l}\text { Triangulary } \\
\text { Index, } \\
\text { units }\end{array}$ & $\begin{array}{l}\text { Before } \\
\text { After } \\
\text { Change }\end{array}$ & $\begin{array}{c}7,4 \pm 0,7^{*} \\
9,2 \pm 0,9^{*} \\
+1,9 \pm 0,3^{\#}\end{array}$ & $\begin{array}{l}7,9 \pm 1,1^{*} \\
8,0 \pm 0,9^{*} \\
+0,1 \pm 0,7\end{array}$ & $\begin{array}{l}10,9 \pm 0,8 \\
8,6 \pm 0,7^{*} \\
-2,2 \pm 0,4^{\#}\end{array}$ & $11,2 \pm 0,3$ \\
\hline $\begin{array}{l}\mathrm{HF} \text {, } \\
\mathrm{msec}^{2}\end{array}$ & $\begin{array}{l}\text { Before } \\
\text { After } \\
\text { Change }\end{array}$ & $\begin{array}{c}234 \pm 100 \\
278 \pm 132 \\
+44 \pm 33 \\
\end{array}$ & $\begin{array}{c}100 \pm 33^{*} \\
105 \pm 30^{*} \\
+5 \pm 32\end{array}$ & $\begin{array}{c}453 \pm 146 \\
275 \pm 92 \\
-178 \pm 68^{\#}\end{array}$ & $413 \pm 76$ \\
\hline $\begin{array}{l}\mathrm{LF}, \\
\mathrm{msec}^{2}\end{array}$ & $\begin{array}{l}\text { Before } \\
\text { After } \\
\text { Change }\end{array}$ & $\begin{array}{c}581 \pm 102 \\
748 \pm 132 \\
+167 \pm 65^{\#}\end{array}$ & $\begin{array}{c}322 \pm 132^{*} \\
328 \pm 118^{*} \\
\quad+6 \pm 42\end{array}$ & $\begin{aligned} 921 & \pm 120 \\
641 & \pm 110 \\
-280 & \pm 119^{\#}\end{aligned}$ & $741 \pm 53$ \\
\hline $\begin{array}{l}\text { VLF, } \\
\text { msec }^{2}\end{array}$ & $\begin{array}{l}\text { Before } \\
\text { After } \\
\text { Change }\end{array}$ & $\begin{array}{c}441 \pm 72^{*} \\
764 \pm 287^{*} \\
+323 \pm 253\end{array}$ & $\begin{array}{c}611 \pm 123^{*} \\
572 \pm 154^{*} \\
-40 \pm 44\end{array}$ & $\begin{array}{l}1005 \pm 57^{*} \\
573 \pm 120^{*} \\
-432 \pm 79^{\#}\end{array}$ & $1495 \pm 118$ \\
\hline $\begin{array}{l}\mathrm{ULF}, \\
\mathrm{msec}^{2}\end{array}$ & $\begin{array}{l}\text { Before } \\
\text { After } \\
\text { Change }\end{array}$ & $\begin{array}{c}71 \pm 22 \\
141 \pm 56 \\
+70 \pm 51\end{array}$ & $\begin{array}{l}172 \pm 50 \\
120 \pm 60 \\
-52 \pm 54\end{array}$ & $\begin{aligned} 170 & \pm 42 \\
70 & \pm 20 \\
-100 & \pm 41^{\#}\end{aligned}$ & $122 \pm 17$ \\
\hline $\begin{array}{l}\text { Bayevskiy's } \\
\text { Activity } \\
\text { Regulatory } \\
\text { Systems } \\
\text { Index, units }\end{array}$ & $\begin{array}{l}\text { Before } \\
\text { After } \\
\text { Change }\end{array}$ & $\begin{array}{l}4,5 \pm 0,8^{*} \\
4,1 \pm 0,7^{*} \\
-0,4 \pm 0,5\end{array}$ & $\begin{array}{l}3,6 \pm 0,9^{*} \\
4,6 \pm 0,9^{*} \\
+1,0 \pm 0,7\end{array}$ & $\begin{array}{l}2,8 \pm 0,5^{*} \\
4,5 \pm 0,5^{*} \\
+1,7 \pm 0,4^{\#}\end{array}$ & $1,5 \pm 0,2$ \\
\hline
\end{tabular}

Table 3

Comparative characteristics of principal Hormonal and Leukocytary markers of General Adaptation Reactions

\begin{tabular}{|c|c|c|c|c|c|}
\hline \multirow{2}{*}{ Variables } & \multirow{2}{*}{ Term } & \multicolumn{3}{|c|}{ Immediate vegetotropic reaction (n) } & \multirow{2}{*}{$\begin{array}{c}\text { Norma- } \\
\text { tives } \\
(n=54)\end{array}$} \\
\hline & & Vagotonic (21) & Neutral (6) & Sympathotonic (27) & \\
\hline $\begin{array}{l}\text { Cortisol, } \\
\mathrm{nM} / \mathrm{L}\end{array}$ & $\begin{array}{l}\text { Before } \\
\text { After } \\
\text { Change }\end{array}$ & $\begin{array}{c}644 \pm 95^{*} \\
526 \pm 64 \\
-118 \pm 87 \\
\end{array}$ & $\begin{array}{l}553 \pm 114 \\
538 \pm 186 \\
-15 \pm 151 \\
\end{array}$ & $\begin{array}{l}496 \pm 40 \\
432 \pm 53 \\
-64 \pm 46 \\
\end{array}$ & $405 \pm 23$ \\
\hline $\begin{array}{l}(\mathrm{Nap} / \mathrm{Kp})^{0,5} \text { as } \\
\text { Mineralo- } \\
\text { corticoid } \\
\text { Activity, units }\end{array}$ & $\begin{array}{l}\text { Before } \\
\text { After } \\
\text { Change }\end{array}$ & $\begin{array}{l}6,64 \pm 0,06^{*} \\
6,58 \pm 0,03^{*} \\
-0,07 \pm 0,05\end{array}$ & $\begin{array}{l}6,47 \pm 0,07^{*} \\
6,45 \pm 0,09^{*} \\
-0,03 \pm 0,07\end{array}$ & $\begin{array}{l}6,53 \pm 0,02^{*} \\
6,54 \pm 0,02^{*} \\
+0,01 \pm 0,02\end{array}$ & $5,65 \pm 0,11$ \\
\hline $\begin{array}{l}\text { Triiodo- } \\
\text { thyronine, } \\
\text { nM/L }\end{array}$ & $\begin{array}{l}\text { Before } \\
\text { After } \\
\text { Change }\end{array}$ & $\begin{array}{l}1,87 \pm 0,04^{*} \\
1,84 \pm 0,04^{*} \\
-0,03 \pm 0,02\end{array}$ & $\begin{array}{l}1,73 \pm 0,06^{*} \\
1,81 \pm 0,06^{*} \\
+0,07 \pm 0,11\end{array}$ & $\begin{array}{l}1,90 \pm 0,04^{*} \\
1,89 \pm 0,03^{*} \\
-0,01 \pm 0,03\end{array}$ & $2,20 \pm 0,09$ \\
\hline $\begin{array}{c}\text { Testosterone, } \\
\text { nM/L }\end{array}$ & $\begin{array}{l}\text { Before } \\
\text { After } \\
\text { Change }\end{array}$ & $\begin{array}{c}24,2 \pm 1,9 \\
27,1 \pm 2,0 \\
+2,9 \pm 1,4^{\#}\end{array}$ & $\begin{array}{l}27,2 \pm 3,9 \\
20,4 \pm 3,6 \\
-6,8 \pm 4,7\end{array}$ & $\begin{array}{l}28,2 \pm 1,5 \\
24,7 \pm 1,7 \\
-3,4 \pm 2,3\end{array}$ & $25,2 \pm 1,2$ \\
\hline
\end{tabular}




\begin{tabular}{|c|c|c|c|c|c|}
\hline Popovych's & Before & $0,84 \pm 0,12^{*}$ & $0,98 \pm 0,22^{*}$ & $1,18 \pm 0,12^{*}$ & $1,70 \pm 0,04$ \\
Leukocytary & After & $1,00 \pm 0,12^{*}$ & $1,47 \pm 0,31$ & $1,17 \pm 0,12^{*}$ & \\
Adaptation & Change & $+0,16 \pm 0,10$ & $+0,49 \pm 0,31$ & $-0,01 \pm 0,15$ & \\
Index, & & & & & \\
points & & & & & \\
\hline Popovych's & Before & $0,56 \pm 0,17^{*}$ & $0,34 \pm 0,14^{*}$ & $0,19 \pm 0,04^{*}$ & 0 \\
Leukocytary & After & $0,27 \pm 0,08^{*}$ & $0,58 \pm 0,34$ & $0,16 \pm 0,03^{*}$ & \\
Strain Index, & Change & $-0,29 \pm 0,14^{\#}$ & $+0,24 \pm 0,23$ & $-0,03 \pm 0,05$ & \\
units & & & & & \\
\hline
\end{tabular}

Among the parameters of Phagocytosis, no significant changes were detected in all variants of the vegetotropic reaction (Table 4).

Among the parameters of Cellular Immunity, only T-cytolytic Lymphocytes level has elevated significantly by sympathetic response (Table 5).

Table 4

Comparative characteristics of parameters of Phagocytosis

\begin{tabular}{|c|c|c|c|c|c|}
\hline \multirow{2}{*}{ Variables } & Term & \multicolumn{3}{|c|}{ Immediate vegetotropic reaction (n) } & \multirow{2}{*}{$\begin{array}{c}\text { Norma- } \\
\text { tives } \\
(\boldsymbol{n = 5 4 )}\end{array}$} \\
\cline { 3 - 5 } & & Vagotonic (21) & Neutral (6) & Sympathotonic (27) & \\
\hline Total & Before & $6,36 \pm 0,41$ & $6,21 \pm 0,24$ & $6,11 \pm 0,27$ & $5,78 \pm 0,33$ \\
Leukocytes, & After & $6,68 \pm 0,39$ & $6,21 \pm 0,45$ & $6,35 \pm 0,34$ & \\
$10^{\%} / \mathrm{L}$ & Change & $+0,31 \pm 0,57$ & $0,00 \pm 0,50$ & $+0,24 \pm 0,39$ & \\
\hline Neutrophiles, & Before & $60,1 \pm 1,3^{*}$ & $53,6 \pm 2,8$ & $59,7 \pm 1,2$ & $56,5 \pm 1,8$ \\
$\%$ & After & $59,3 \pm 1,3$ & $53,6 \pm 2,9$ & $58,9 \pm 1,3$ & \\
& Change & $-0,8 \pm 1,2$ & $0,0 \pm 2,7$ & $-0,8 \pm 0,9$ & \\
\hline Phagocytose & Before & $87,1 \pm 1,2^{*}$ & $85,7 \pm 1,3^{*}$ & $86,8 \pm 0,9^{*}$ & $80,0 \pm 1,5$ \\
Index, & After & $86,7 \pm 0,9^{*}$ & $86,0 \pm 1,2^{*}$ & $86,7 \pm 0,9^{*}$ & \\
$\%$ & Change & $-0,4 \pm 1,2$ & $+0,3 \pm 1,8$ & $-0,1 \pm 0,9$ & \\
\hline Microbial & Before & $14,4 \pm 0,6$ & $14,5 \pm 1,3$ & $14,1 \pm 0,5$ & $14,4 \pm 0,8$ \\
Count, & After & $14,4 \pm 0,6$ & $15,5 \pm 1,9$ & $13,9 \pm 0,6$ & \\
Microbas/ & Change & $0,0 \pm 0,6$ & $+1,0 \pm 1,6$ & $-0,3 \pm 0,5$ & \\
Phagocyte & & & & & \\
\hline Killing Index, & Before & $32 \pm 4$ & $33 \pm 4$ & $38 \pm 2$ & \\
\% & After & $31 \pm 3^{*}$ & $31 \pm 5$ & $35 \pm 2$ & \\
& Change & $0 \pm 2$ & $-2 \pm 4$ & $-3 \pm 2$ & \\
\hline Bactericidal & Before & $14,6 \pm 1,7$ & $13,2 \pm 1,5$ & $16,6 \pm 1,2$ & $15,0 \pm 0,9$ \\
Capacity, & After & $15,3 \pm 2,0$ & $12,7 \pm 1,9$ & $15,0 \pm 1,2$ & \\
$10^{9}$ Microbas/L & Change & $+0,8 \pm 1,8$ & $-0,5 \pm 1,5$ & $-1,6 \pm 1,3$ & \\
\hline
\end{tabular}

Table 5

Comparative characteristics of parameters of Cellular Immunity

\begin{tabular}{|c|c|c|c|c|c|}
\hline \multirow{2}{*}{ Variables } & \multirow{2}{*}{ Term } & \multicolumn{3}{|c|}{ Immediate vegetotropic reaction (n) } & \multirow{2}{*}{$\begin{array}{c}\text { Norma- } \\
\text { tives } \\
(n=54)\end{array}$} \\
\hline & & Vagotonic (21) & Neutral (6) & Sympathotonic (27) & \\
\hline $\begin{array}{c}\text { Total } \\
\text { Lymphocytes, } \\
10^{9} / \mathrm{L}\end{array}$ & $\begin{array}{l}\text { Before } \\
\text { After } \\
\text { Change }\end{array}$ & $\begin{array}{l}2,31 \pm 0,13^{*} \\
2,26 \pm 0,10^{*} \\
-0,05 \pm 0,12\end{array}$ & $\begin{array}{l}2,23 \pm 0,07^{*} \\
2,24 \pm 0,16 \\
0,00 \pm 0,13\end{array}$ & $\begin{array}{l}2,23 \pm 0,10^{*} \\
2,26 \pm 0,09^{*} \\
+0,03 \pm 0,11\end{array}$ & $\begin{array}{c}1,96 \pm \\
0,04\end{array}$ \\
\hline $\begin{array}{c}\text { "Active" } \\
\text { T-Lymphocytes, } \\
\%\end{array}$ & $\begin{array}{l}\text { Before } \\
\text { After } \\
\text { Change }\end{array}$ & $\begin{array}{c}26,6 \pm 1,1^{*} \\
25,4 \pm 0,6^{*} \\
-1,2 \pm 0,7\end{array}$ & $\begin{array}{l}26,8 \pm 1,5 \\
27,2 \pm 2,0 \\
+0,4 \pm 0,6\end{array}$ & $\begin{array}{c}25,6 \pm 0,6^{*} \\
25,4 \pm 0,8^{*} \\
-0,2 \pm 0,7\end{array}$ & $29,6 \pm 0,8$ \\
\hline $\begin{array}{c}\text { Theophilline } \\
\text { resistance } \\
\text { T-Lymphocytes, \% }\end{array}$ & $\begin{array}{l}\text { Before } \\
\text { After } \\
\text { Change }\end{array}$ & $\begin{array}{l}21,9 \pm 0,8^{*} \\
22,1 \pm 0,9^{*} \\
+0,2 \pm 1,0\end{array}$ & $\begin{array}{l}30,0 \pm 4,5 \\
28,7 \pm 3,1 \\
-1,3 \pm 3,1\end{array}$ & $\begin{array}{l}21,9 \pm 1,2^{*} \\
23,1 \pm 1,2^{*} \\
+1,2 \pm 1,2\end{array}$ & $33,2 \pm 1,2$ \\
\hline
\end{tabular}



https://

\begin{tabular}{|c|c|c|c|c|c|}
\hline T-helper & Before & $23,9 \pm 0,6^{*}$ & $27,5 \pm 1,9$ & $23,6 \pm 0,8^{*}$ & $29,1 \pm 1,0$ \\
Lymphocytes, & After & $23,5 \pm 0,4^{*}$ & $26,9 \pm 1,7$ & $23,0 \pm 0,8^{*}$ & \\
\% & Change & $-0,4 \pm 0,8$ & $-0,6 \pm 1,3$ & $-0,6 \pm 0,8$ & \\
\hline Theophilline & Before & $20,6 \pm 1,1$ & $16,9 \pm 3,5$ & $18,2 \pm 1,1^{*}$ & $20,9 \pm 0,4$ \\
sensitive & After & $18,5 \pm 1,3$ & $14,1 \pm 3,6$ & $19,4 \pm 1,2$ & \\
T-Lymphocytes, \% & Change & $-2,2 \pm 1,7$ & $-2,8 \pm 5,6$ & $+1,2 \pm 1,5$ & \\
\hline CD8+CD3+ & Before & $27,9 \pm 0,5^{*}$ & $26,2 \pm 1,0$ & $27,1 \pm 0,6^{*}$ & $24,8 \pm 0,5$ \\
T-cytolytic & After & $26,8 \pm 0,8^{*}$ & $24,6 \pm 1,5$ & $28,3 \pm 0,8^{*}$ & \\
Lymphocytes, \% & Change & $-1,1 \pm 0,8$ & $-1,7 \pm 1,9$ & $+1,3 \pm 0,6^{*}$ & \\
\hline CD16+ NK- & Before & $11,4 \pm 0,8^{*}$ & $11,3 \pm 1,1^{*}$ & $11,7 \pm 0,5^{*}$ & $16,4 \pm 0,8$ \\
Lymphocytes, & After & $12,2 \pm 0,7^{*}$ & $11,2 \pm 1,5^{*}$ & $11,5 \pm 0,7^{*}$ & \\
$\%$ & Change & $+0,8 \pm 0,5$ & $-0,1 \pm 1,2$ & $-0,2 \pm 0,7$ & \\
\hline 0-Lymphocytes, & Before & $14,2 \pm 1,2^{*}$ & $12,9 \pm 4,0$ & $16,2 \pm 1,7^{*}$ & $8,0 \pm 0,8$ \\
$\%$ & After & $16,1 \pm 1,5^{*}$ & $14,4 \pm 5,2$ & $16,5 \pm 2,0^{*}$ & \\
& Change & $+1,9 \pm 1,7$ & $+1,5 \pm 4,1$ & $+0,3 \pm 2,3$ & \\
\hline
\end{tabular}

None of the parameters of Humoral Immunity has changed significantly for all variants of the vegetotropic reaction (Table 6).

Table 6

Comparative characteristics of parameters of Humoral Immunity

\begin{tabular}{|c|c|c|c|c|c|}
\hline \multirow{2}{*}{ Variables } & \multirow{2}{*}{ Term } & \multicolumn{3}{|c|}{ Immediate vegetotropic reaction (n) } & \multirow{2}{*}{$\begin{array}{c}\text { Norma- } \\
\text { tives } \\
(n=54)\end{array}$} \\
\hline & & Vagotonic (21) & Neutral (6) & Sympathotonic (27) & \\
\hline $\begin{array}{c}\text { CD19+ B-Lym- } \\
\text { phocytes, } \\
\%\end{array}$ & $\begin{array}{l}\text { Before } \\
\text { After } \\
\text { Change }\end{array}$ & $\begin{array}{l}22,6 \pm 0,9 \\
21,3 \pm 1,0 \\
-1,3 \pm 1,1\end{array}$ & $\begin{array}{l}22,0 \pm 2,7 \\
23,0 \pm 2,2 \\
+1,0 \pm 3,0\end{array}$ & $\begin{array}{l}21,5 \pm 0,9 \\
20,8 \pm 1,0 \\
-0,8 \pm 1,4\end{array}$ & $21,7 \pm 0,8$ \\
\hline $\begin{array}{l}\text { Immuno- } \\
\text { globulins } \mathrm{M} \text {, } \\
\mathrm{g} / \mathrm{L}\end{array}$ & $\begin{array}{l}\text { Before } \\
\text { After } \\
\text { Change }\end{array}$ & $\begin{array}{l}1,52 \pm 0,09^{*} \\
1,61 \pm 0,12^{*} \\
+0,10 \pm 0,11\end{array}$ & $\begin{array}{r}1,45 \pm 0,29 \\
1,20 \pm 0,23 \\
-0,25 \pm 0,24\end{array}$ & $\begin{array}{l}1,36 \pm 0,09^{*} \\
1,48 \pm 0,08^{*} \\
+0,12 \pm 0,08\end{array}$ & $1,15 \pm 0,05$ \\
\hline $\begin{array}{l}\text { Immuno- } \\
\text { globulins G, } \\
\text { g/L }\end{array}$ & $\begin{array}{l}\text { Before } \\
\text { After } \\
\text { Change }\end{array}$ & $\begin{array}{l}12,9 \pm 1,3 \\
11,6 \pm 1,3 \\
-1,4 \pm 1,4\end{array}$ & $\begin{array}{l}15,0 \pm 3,6 \\
16,5 \pm 2,8 \\
+1,6 \pm 1,7\end{array}$ & $\begin{array}{l}12,7 \pm 1,3 \\
11,7 \pm 1,4 \\
-1,0 \pm 1,0\end{array}$ & $11,5 \pm 0,4$ \\
\hline $\begin{array}{c}\text { Immuno- } \\
\text { globulins A, } \\
\mathrm{g} / \mathrm{L}\end{array}$ & $\begin{array}{l}\text { Before } \\
\text { After } \\
\text { Change }\end{array}$ & $\begin{array}{l}1,40 \pm 0,07^{*} \\
1,37 \pm 0,13^{*} \\
-0,03 \pm 0,14\end{array}$ & $\begin{array}{c}1,19 \pm 0,15^{*} \\
1,60 \pm 0,43 \\
+0,40 \pm 0,41\end{array}$ & $\begin{array}{l}1,27 \pm 0,05^{*} \\
1,26 \pm 0,06^{*} \\
-0,01 \pm 0,23\end{array}$ & $1,90 \pm 0,06$ \\
\hline $\begin{array}{l}\text { Circulating } \\
\text { Immune } \\
\text { Complexes, } \\
\text { units }\end{array}$ & $\begin{array}{l}\text { Before } \\
\text { After } \\
\text { Change }\end{array}$ & $\begin{array}{c}74 \pm 24 \\
52 \pm 11 \\
-22 \pm 20\end{array}$ & $\begin{array}{c}42 \pm 4 \\
35 \pm 5^{*} \\
-7 \pm 5\end{array}$ & $\begin{array}{c}38 \pm 3^{*} \\
33 \pm 3^{*} \\
-4 \pm 3\end{array}$ & $54 \pm 5$ \\
\hline
\end{tabular}

Since the number of registered EEG parameters is 164, we limited its number to only 15, and then their changes have been proved to be characteristic of the vegetotropic reactions (Table 7). As it can be seen, the vagotonic reaction is associated with the significant increase in the B-rhythm Index and the decrease in its SPD in locus T5. Instead, the sympathetic response is accompanied by the decrease in the Frequency of a-rhythm and its SPD in the loci T5 and F7 as well as by the increase in the Entropy of SPD in locus O1.

Now let's turn to the presentation of the results of the already mentioned discriminant analysis which was conducted to identify the parameters of the neuroendocrine-immune complex, among the totality of changes three variants of the vegetotropic reaction differ significantly among themselves, that is, they are discriminational [13].

The program (forward stepwise) included 27 parameters (variables) in the model. In addition the Baevskiy's Stress Index, 3 parameters of HRV, 15 of 
EEG and 7 of Immunity as well as Testosterone were identified as recognizable (Table 8).

Table 7

Comparative characteristics of selected parameters of EEG

\begin{tabular}{|c|c|c|c|c|c|}
\hline \multirow{2}{*}{ Variables } & \multirow{2}{*}{ Term } & \multicolumn{3}{|c|}{ Immediate vegetotropic reaction (n) } & \multirow{2}{*}{$\begin{array}{c}\text { Norma- } \\
\text { tives } \\
(n=54) \\
\end{array}$} \\
\hline & & Vagotonic (21) & Neutral (6) & Sympathotonic (27) & \\
\hline $\begin{array}{c}\text { T5-a SPD } \\
\mu \mathrm{V}^{2} / \mathrm{Hz}\end{array}$ & $\begin{array}{l}\text { Before } \\
\text { After } \\
\text { Change }\end{array}$ & $\begin{array}{c}75 \pm 22 \\
80 \pm 22 \\
+4 \pm 9\end{array}$ & $\begin{array}{c}114 \pm 59 \\
163 \pm 108 \\
+50 \pm 51\end{array}$ & $\begin{array}{c}122 \pm 28 \\
97 \pm 21 \\
-25 \pm 11^{\#}\end{array}$ & $134 \pm 16$ \\
\hline $\begin{array}{c}\text { T5-a SPD, } \\
\%\end{array}$ & $\begin{array}{l}\text { Before } \\
\text { After } \\
\text { Change }\end{array}$ & $\begin{array}{l}26,8 \pm 3,7 \\
29,6 \pm 4,2 \\
+2,8 \pm 2,4\end{array}$ & $\begin{array}{c}38,1 \pm 8,3 \\
37,0 \pm 10,5 \\
-1,1 \pm 6,5\end{array}$ & $\begin{array}{l}35,5 \pm 3,9 \\
30,3 \pm 3,1 \\
-5,2 \pm 2,0^{\#}\end{array}$ & $36,8 \pm 2,3$ \\
\hline $\begin{array}{c}\text { T5-8 SPD } \\
\%\end{array}$ & $\begin{array}{c}\text { Before } \\
\text { After } \\
\text { Change }\end{array}$ & $\begin{array}{c}46 \pm 4 \\
37 \pm 4 \\
-8,4 \pm 3,0^{\#}\end{array}$ & $\begin{array}{c}35 \pm 8 \\
33 \pm 10 \\
-1,4 \pm 4,6\end{array}$ & $\begin{array}{c}39 \pm 4 \\
39 \pm 4 \\
-0,5 \pm 3,7\end{array}$ & $36,8 \pm 2,4$ \\
\hline $\begin{array}{c}\text { F7- } \theta \text { SPD } \\
\mu \mathrm{V}^{2} / \mathrm{Hz}\end{array}$ & $\begin{array}{l}\text { Before } \\
\text { After } \\
\text { Change }\end{array}$ & $\begin{array}{l}14,5 \pm 3,2 \\
14,0 \pm 2,3 \\
-0,5 \pm 3,0\end{array}$ & $\begin{array}{l}12,2 \pm 3,0 \\
11,1 \pm 3,5 \\
-1,1 \pm 4,7\end{array}$ & $\begin{array}{l}13,8 \pm 1,7 \\
20,4 \pm 3,8 \\
+6,6 \pm 3,5\end{array}$ & $16,0 \pm 1,6$ \\
\hline $\begin{array}{c}\text { Fp1- } \delta \text { SPD } \\
\%\end{array}$ & $\begin{array}{l}\text { Before } \\
\text { After } \\
\text { Change }\end{array}$ & $\begin{array}{l}21,8 \pm 3,6 \\
23,5 \pm 4,7 \\
+1,7 \pm 5,4\end{array}$ & $\begin{array}{c}10,6 \pm 1,7 \\
37,2 \pm 16,1 \\
+26,6 \pm 16,3\end{array}$ & $\begin{array}{l}18,0 \pm 2,3 \\
22,1 \pm 3,7 \\
+4,1 \pm 4,1\end{array}$ & $18,9 \pm 1,4$ \\
\hline $\begin{array}{c}\text { Frequency } \\
\text { of a-rhythm, } \\
\mathrm{Hz}\end{array}$ & $\begin{array}{c}\text { Before } \\
\text { After } \\
\text { Change }\end{array}$ & $\begin{array}{l}10,64 \pm 0,19 \\
10,48 \pm 0,22 \\
-0,17 \pm 0,23\end{array}$ & $\begin{array}{l}10,58 \pm 0,27 \\
11,08 \pm 0,52 \\
+0,50 \pm 0,34\end{array}$ & $\begin{array}{c}10,52 \pm 0,18 \\
10,48 \pm 0,15 \\
-0,24 \pm 0,06^{\#}\end{array}$ & $10,43 \pm 0,08$ \\
\hline $\begin{array}{c}\text { Asymmetry } \\
\text { of B-rhythm, } \\
\%\end{array}$ & $\begin{array}{c}\text { Before } \\
\text { After } \\
\text { Change }\end{array}$ & $\begin{array}{c}24 \pm 5 \\
17 \pm 2 \\
-7,4 \pm 4,9\end{array}$ & $\begin{array}{c}28 \pm 10 \\
28 \pm 10 \\
-0,3 \pm 5,6\end{array}$ & $\begin{array}{c}16 \pm 2 \\
17 \pm 3 \\
+0,4 \pm 2,2\end{array}$ & $20 \pm 1,5$ \\
\hline $\begin{array}{c}\text { F8-6 SPD } \\
\mu V^{2} / H z\end{array}$ & $\begin{array}{l}\text { Before } \\
\text { After } \\
\text { Change }\end{array}$ & $\begin{array}{c}77 \pm 22 \\
66 \pm 20 \\
-11 \pm 18\end{array}$ & $\begin{array}{l}43 \pm 15 \\
46 \pm 13 \\
+3 \pm 6\end{array}$ & $\begin{array}{c}66 \pm 14 \\
51 \pm 7 \\
-15 \pm 12\end{array}$ & $69 \pm 9$ \\
\hline $\begin{array}{l}\text { F4- } \delta \text { SPD, } \\
\mu \mathrm{V}_{2} / \mathrm{Hz}\end{array}$ & $\begin{array}{c}\text { Before } \\
\text { After } \\
\text { Change }\end{array}$ & $\begin{array}{c}150 \pm 70 \\
85 \pm 14 \\
-65 \pm 65 \\
\end{array}$ & $\begin{array}{c}54 \pm 14 \\
119 \pm 69 \\
+65 \pm 78 \\
\end{array}$ & $\begin{array}{c}77 \pm 14 \\
229 \pm 141 \\
+152 \pm 143 \\
\end{array}$ & $89 \pm 9$ \\
\hline $\begin{array}{c}\text { F7- } \alpha \text { SPD } \\
\%\end{array}$ & $\begin{array}{c}\text { Before } \\
\text { After } \\
\text { Change }\end{array}$ & $\begin{array}{c}25 \pm 3 \\
28 \pm 4 \\
+3,2 \pm 2,6\end{array}$ & $\begin{array}{c}29 \pm 7 \\
33 \pm 8 \\
+4,1 \pm 7,3\end{array}$ & $\begin{array}{c}32 \pm 3 \\
26 \pm 3 \\
-6,4 \pm 2,3^{\#}\end{array}$ & $31,7 \pm 2,0$ \\
\hline $\begin{array}{c}\text { Fp1-a SPD } \\
\%\end{array}$ & $\begin{array}{l}\text { Before } \\
\text { After } \\
\text { Change }\end{array}$ & $\begin{array}{c}34 \pm 4 \\
36 \pm 5 \\
+2,0 \pm 2,8\end{array}$ & $\begin{array}{c}41 \pm 9 \\
28 \pm 11 \\
-13 \pm 9\end{array}$ & $\begin{array}{c}40 \pm 3 \\
36 \pm 3 \\
-3,6 \pm 2,5\end{array}$ & $44,0 \pm 2,2$ \\
\hline $\begin{array}{c}\text { Index of } \\
\text { B-rhythm, } \\
\%\end{array}$ & $\begin{array}{c}\text { Before } \\
\text { After } \\
\text { Change }\end{array}$ & $\begin{array}{c}76,1 \pm 6,0 \\
88,6 \pm 2,0 \\
+12,5 \pm 6,0^{\#}\end{array}$ & $\begin{array}{l}91,3 \pm 5,7 \\
94,2 \pm 2,4 \\
+2,8 \pm 5,8\end{array}$ & $\begin{array}{l}92,0 \pm 2,8 \\
89,9 \pm 3,0 \\
-2,0 \pm 4,0\end{array}$ & $87,9 \pm 1,8$ \\
\hline $\begin{array}{l}\text { Entropy } \\
\text { of SPD } \\
\text { in O1 }\end{array}$ & $\begin{array}{l}\text { Before } \\
\text { After } \\
\text { Change }\end{array}$ & $\begin{array}{c}0,74 \pm 0,04 \\
0,76 \pm 0,04 \\
+0,03 \pm 0,04\end{array}$ & $\begin{array}{c}0,78 \pm 0,05 \\
0,70 \pm 0,06 \\
-0,08 \pm 0,06\end{array}$ & $\begin{array}{c}0,71 \pm 0,03 \\
0,76 \pm 0,03 \\
+0,05 \pm 0,0^{2 \#}\end{array}$ & $0,68 \pm 0,02$ \\
\hline $\begin{array}{l}\text { Entropy } \\
\text { of SPD } \\
\text { in F4 }\end{array}$ & $\begin{array}{c}\text { Before } \\
\text { After } \\
\text { Change }\end{array}$ & $\begin{array}{c}0,82 \pm 0,03 \\
0,86 \pm 0,02 \\
+0,03 \pm 0,03\end{array}$ & $\begin{array}{c}0,87 \pm 0,05 \\
0,83 \pm 0,04 \\
-0,04 \pm 0,04\end{array}$ & $\begin{array}{l}0,85 \pm 0,02 \\
0,85 \pm 0,03 \\
0,00 \pm 0,03\end{array}$ & $0,83 \pm 0,01$ \\
\hline $\begin{array}{c}\text { Entropy } \\
\text { of SPD } \\
\text { in P4 }\end{array}$ & $\begin{array}{c}\text { Before } \\
\text { After } \\
\text { Change }\end{array}$ & $\begin{array}{c}0,84 \pm 0,03 \\
0,78 \pm 0,03 \\
-0,06 \pm 0,02^{\#}\end{array}$ & $\begin{array}{c}0,83 \pm 0,04 \\
0,79 \pm 0,05 \\
-0,05 \pm 0,05\end{array}$ & $\begin{array}{c}0,79 \pm 0,03 \\
0,80 \pm 0,02 \\
+0,01 \pm 0,02\end{array}$ & $0,76 \pm 0,01$ \\
\hline
\end{tabular}



https://

The program (forward stepwise) included 27 parameters (variables) in the model. In addition the Baevskiy's Stress Index, 3 parameters of HRV, 15 of EEG and 7 of Immunity as well as Testosterone were identified as recognizable (Table 8).

Table 8

\section{Discriminant Function Analysis Summary}

Step 27, N of variables in model: 27; Grouping: 3 groups

Wilks' La mbda: 0,0179; approximately. $\mathrm{F}_{(54)}=6,0 ; p<10^{-6}$

\begin{tabular}{|c|c|c|c|c|c|}
\hline Variables currently in model & $\begin{array}{l}\text { Wilks' } \\
\Lambda\end{array}$ & $\begin{array}{c}\text { Partial } \\
\Lambda\end{array}$ & F-remove & p-level & Tolerancy \\
\hline Baevskiy's Stress Index, ln units &, 023 & ,788 & 3,4 & 051 &, 125 \\
\hline Triangulary Index, units & ,021 & ,857 & 2,1 &, 145 & ,348 \\
\hline $\mathrm{T} 5-\alpha \mathrm{SPD}, \mu \mathrm{V}^{2} / \mathrm{Hz}$ & ,022 & ,808 & 3,0 & ,070 & ,379 \\
\hline T5-8 SPD, \% &, 027 &, 657 & 6,5 &, 005 &, 220 \\
\hline F7- $\theta$ SPD, $\mu V^{2} / \mathrm{Hz}$ &, 026 &, 683 & 5,8 &, 009 &, 259 \\
\hline Fp1- $\delta$ SPD, \% & ,019 & ,957 & ,6 &, 579 & ,103 \\
\hline Frequency of a-rhythm, $\mathrm{Hz}$ &, 031 &, 579 & 9,1 & ,001 & ,298 \\
\hline Stub Neutrophils, \% &, 024 & ,755 & 4,1 &, 030 & ,313 \\
\hline 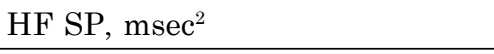 &, 021 & ,868 & 1,9 &, 170 &, 520 \\
\hline $\begin{array}{l}\text { Popovych's Adaptation Index, } \\
\text { points }\end{array}$ &, 024 &, 748 & 4,2 &, 027 &, 025 \\
\hline Asymmetry of B-rhythm, \% &, 022 & ,830 & 2,6 & ,098 &, 370 \\
\hline CD8+CD3+ T-cytolyc Lymphoc., \% &, 021 & ,835 & 2,5 &, 106 & ,365 \\
\hline Entropy of SPD in $\mathrm{O} 1$ &, 026 &, 700 & 5,4 &, 012 &, 294 \\
\hline Popovych's Strain Index, units &, 030 &, 605 & 8,2 &, 002 &, 256 \\
\hline Testosterone, $\mathrm{nM} / \mathrm{L}$ &, 021 &, 859 & 2,1 &, 149 &, 030 \\
\hline F8-6 SPD, $\mu V^{2} / \mathrm{Hz}$ &, 022 &, 820 & 2,7 &, 084 &, 424 \\
\hline F4- $\delta$ SPD, $\mu V^{2} / \mathrm{Hz}$ &, 018 &, 984 & 0,2 & ,816 & ,215 \\
\hline F7-a SPD, \% &, 039 &, 459 & 14,7 &, 000 & ,089 \\
\hline Fp1-a SPD, \% &, 029 &, 621 & 7,6 &, 003 &, 059 \\
\hline T5-a SPD, \% &, 021 & ,832 & 2,5 &, 100 & ,317 \\
\hline LF SP, $\mathrm{msec}^{2}$ &, 022 & ,808 & 3,0 &, 069 &, 287 \\
\hline IgA, g/L &, 022 &, 806 & 3,0 &, 067 &, 261 \\
\hline $\operatorname{IgM}, \mathrm{g} / \mathrm{L}$ &, 020 &, 895 & 1,5 & ,249 & ,435 \\
\hline Entropy of SPD in F4 &, 023 & ,793 & 3,3 &, 055 &, 168 \\
\hline "Active" T-Lymphocytes, \% &, 021 &, 851 & 2,2 &, 134 & ,302 \\
\hline Entropy of SPD in P4 &, 020 &, 903 & 1,3 &, 279 & ,403 \\
\hline Index of B-rhythm, \% &, 019 &, 920 & 1,1 & ,355 &, 608 \\
\hline
\end{tabular}

The recognition information contained in the listed variables is condensed in two discriminant roots, $62 \%$ in the first and $38 \%$ in the second. The values of individual roots were calculated by adding products of discriminant variables to their Raw Coefficients plus the Constant (Table 9) makes it possible to visualize each vegetotropic reaction together with its neuroendocrine-immune accompaniment in a two-dimensional information space of radicals (Fig. 1). 
Table 9

\section{Summary of Stepwise Analysis and Coefficients and Constants for Canonical Variables}

\begin{tabular}{|c|c|c|c|c|c|c|c|c|c|}
\hline \multirow[t]{2}{*}{$\begin{array}{l}\text { Variables currently } \\
\text { in the model }\end{array}$} & \multicolumn{5}{|c|}{ Parameters of Wilks' Statistics } & \multicolumn{2}{|c|}{$\begin{array}{c}\text { Standardized } \\
\text { Coefficients }\end{array}$} & \multicolumn{2}{|c|}{$\begin{array}{c}\text { Raw } \\
\text { Coefficients }\end{array}$} \\
\hline & $\begin{array}{c}\text { F to } \\
\text { enter }\end{array}$ & $\begin{array}{c}\text { p- } \\
\text { level }\end{array}$ & $\Lambda$ & $\begin{array}{c}\text { F- } \\
\text { value }\end{array}$ & $\begin{array}{c}\text { p- } \\
\text { level }\end{array}$ & $\begin{array}{c}\text { Root } \\
1\end{array}$ & $\begin{array}{c}\text { Root } \\
2\end{array}$ & $\begin{array}{c}\text { Root } \\
1\end{array}$ & $\begin{array}{c}\text { Root } \\
\quad 2\end{array}$ \\
\hline $\begin{array}{l}\text { Baevskiy's Stress Index, } \\
\text { ln un. }\end{array}$ & 33,3 & $10^{-6}$ & ,434 & 33,3 & $10^{-6}$ & $-1,201$ & ,699 & $-2,413$ & 1,405 \\
\hline Triangulary Index, units & 5,2 & ,009 & ,360 & 16,7 & $10^{-6}$ & ,627 &,- 269 & ,332 &,- 143 \\
\hline T5- $\alpha \mathrm{SPD}, \mu \mathrm{V}^{2} / \mathrm{Hz}$ & 2,6 & ,088 & ,326 & 12,3 & $10^{-6}$ &,- 277 &, 725 &,- 004 &, 011 \\
\hline T5-6 SPD, \% & 2,6 & ,082 & ,293 & 10,2 & $10^{-6}$ & $-1,097$ & ,761 &,- 066 & 046 \\
\hline F7- $\theta \mathrm{SPD}, \mu \mathrm{V}^{2} / \mathrm{Hz}$ & 4,9 &, 012 & ,243 & 9,7 & $10^{-6}$ & $-1,084$ &, 454 &,- 068 & ,028 \\
\hline Fp1- $\delta$ SPD, \% & 3,8 &, 029 & ,208 & 9,1 & $10^{-6}$ &,- 427 &,- 549 &,- 017 &,- 022 \\
\hline Frequency of $\alpha-r h y t h m, \mathrm{~Hz}$ & 3,1 &, 055 & , 183 & 8,6 & $10^{-6}$ &,- 002 & 1,301 &,- 001 & 1,087 \\
\hline Stub Neutrophils, \% & 2,5 &, 097 &, 165 & 8,1 & $10^{-6}$ &, 085 &,- 964 &, 045 &,- 518 \\
\hline HF SP, $\mathrm{msec}^{2}$ & 2,3 &, 113 & , 149 & 7,6 & $10^{-6}$ &, 534 &,- 012 & ,00196 & $-45 \cdot 10^{-6}$ \\
\hline Popovych's Adaptation Index & 2,7 &, 080 &, 132 & 7,4 & $10^{-6}$ & $-1,274$ & 3,228 & $-1,911$ & 4,841 \\
\hline Asymmetry of B-rhythm, \% & 2,6 &, 084 &, 117 & 7,2 & $10^{-6}$ &,- 306 &, 671 &,- 018 &, 040 \\
\hline CD8+CD3+ T-c Lymphocytes & 3,7 &, 034 & 099 & 7,3 & $10^{-6}$ &,- 703 &,- 111 &,- 192 &,- 030 \\
\hline Entropy of SPD in $\mathrm{O} 1$ & 2,1 &, 141 & ,089 & 7,0 & $10^{-6}$ &,- 432 & $-1,013$ & $-2,803$ & $-6,571$ \\
\hline $\begin{array}{l}\text { Popovych's Strain Index, } \\
\text { units }\end{array}$ & 2,1 &, 135 &, 080 & 6,9 & $10^{-6}$ &, 055 & 1,359 &, 120 & 2,939 \\
\hline Testosterone, nM/L & 1,8 &, 174 &, 073 & 6,7 & $10^{-6}$ & $-1,162$ & 2,058 &,- 115 & ,204 \\
\hline F8-B SPD, $\mu V^{2} / \mathrm{Hz}$ & 2,2 &, 129 & ,065 & 6,6 & $10^{-6}$ & ,630 &,- 291 & ,009 &,- 004 \\
\hline F4- $\delta \mathrm{SPD}, \mu \mathrm{V}^{2} / \mathrm{Hz}$ & 1,8 &, 173 & ,059 & 6,4 & $10^{-6}$ & ,290 & ,002 & $51 \cdot 10^{-5}$ & $3 \cdot 10^{-6}$ \\
\hline F7- $\alpha$ SPD, \% & 1,8 &, 188 &, 054 & 6,3 & $10^{-6}$ &, 610 & 2,625 &, 048 &, 206 \\
\hline Fp1-a SPD, \% & 4,9 &, 014 &, 041 & 6,8 & $10^{-6}$ &,- 558 & $-2,719$ &,- 040 &,- 193 \\
\hline T5- $\alpha$ SPD, \% & 2,0 &, 147 &, 037 & 6,8 & $10^{-6}$ &,- 302 &,- 734 &,- 027 &,- 065 \\
\hline $\mathrm{LF}_{\mathrm{SP}}, \mathrm{msec}^{2}$ & 1,9 &, 168 & ,033 & 6,7 & $10^{-6}$ &,- 039 & ,895 & $-8 \cdot 10^{-5}$ & ,0019 \\
\hline $\operatorname{IgA}, g / L$ & 1,8 &, 181 &, 029 & 6,6 & $10^{-6}$ &, 218 & ,918 &, 365 & 1,538 \\
\hline IgM, g/L & 1,2 &, 305 &, 027 & 6,4 & $10^{-6}$ &, 357 & ,393 &, 755 & ,831 \\
\hline Entropy of SPD in F4 & 1,7 &, 198 &, 024 & 6,4 & $10^{-6}$ &, 842 &, 845 & 5,241 & 5,259 \\
\hline "Active" T-Lymphocytes, \% & 1,7 &, 205 &, 021 & 6,3 & $10^{-6}$ &,- 347 &,- 678 &,- 108 &,- 211 \\
\hline Entropy of SPD in $\mathrm{P} 4$ & 1,3 &, 300 &, 019 & 6,2 & $10^{-6}$ &,- 515 &,- 071 & $-4,929$ &,- 677 \\
\hline Index of B-rhythm, \% & 1,1 &, 355 &, 018 & 6,0 & $10^{-6}$ & ,305 &, 239 &, 013 &, 010 \\
\hline \multicolumn{8}{|c|}{ Constants } & ,2698 &,- 0003 \\
\hline \multicolumn{8}{|c|}{ Discriminant Properties } & $62 \%$ & $38 \%$ \\
\hline
\end{tabular}

It can be seen that all three clusters are delimited so clearly that they do not need to compute Mahalanobis distances between them.

The placement of points of the cluster of the vagotonic reaction in the positive region of the first root reflects the increase (meaningful or as a tendency) in this situation parameters correlate with this root positively and reduce the parameters that are associated with it negatively. Instead, the opposite localization along the axis of the first root of the sympathetic cluster reflects the opposite or less noticable changes of the same parameters (Table 1). 


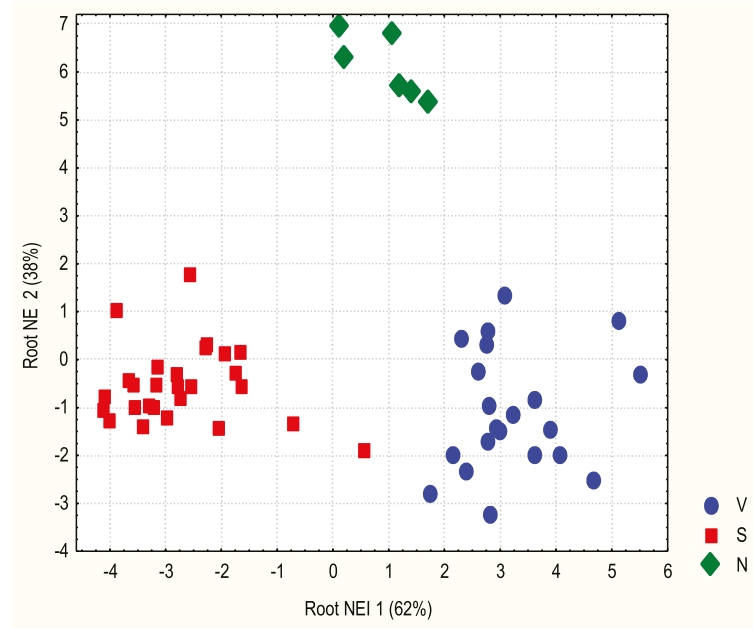

Fig. 1. Localization of individual values of roots of vegetotropic reactions together with its neuroendocrine-immune accompaniment in a two-dimensional information space of radicals

Table 10

Factor Structure Matrix (Correlations Variables - Canonical Roots) and Means of Changes in Variables by Vegetotropic Reaction

\begin{tabular}{|c|c|c|c|c|c|}
\hline \multirow[t]{2}{*}{$\begin{array}{l}\text { Variables currently } \\
\text { in the model }\end{array}$} & \multirow[t]{2}{*}{$\begin{array}{c}\text { Root } \\
1\end{array}$} & \multirow[t]{2}{*}{$\begin{array}{c}\text { Root } \\
2\end{array}$} & \multicolumn{3}{|c|}{$\begin{array}{c}\text { Means of Changes by Vegetotropic } \\
\text { Reaction }\end{array}$} \\
\hline & & & Vagotonic & Neutral & Sympathotonic \\
\hline Triangulary Index, units & 364 &,- 015 & $+1,9 \pm 0,3^{\#}$ & $+0,1 \pm 0,7$ & $-2,2 \pm 0,4^{\#}$ \\
\hline Stub Neutrophils, \% & , 175 & 002 & $+1,13 \pm 0,38^{\#}$ & $+0,42 \pm 0,86$ & $-0,80 \pm 0,37^{\#}$ \\
\hline LF SP, $\mathrm{msec}^{2}$ &, 156 &, 003 & $+167 \pm 65^{\#}$ & $+6 \pm 42$ & $-280 \pm 119^{\#}$ \\
\hline HF SP, $\mathrm{msec}^{2}$ & , 139 & 024 & $+44 \pm 33$ & $+5 \pm 32$ & $-178 \pm 68^{\#}$ \\
\hline F7- $\alpha$ SPD, \% & 131 & ,052 & $+3,2 \pm 2,6$ & $+4,1 \pm 7,3$ & $-6,4 \pm 2,3^{\#}$ \\
\hline T5-a SPD, \% &, 118 &,- 010 & $+2,8 \pm 2,4$ & $-1,1 \pm 6,5$ & $-5,2 \pm 2,0^{\#}$ \\
\hline T5- $\alpha$ SPD, $\mu V^{2} / \mathrm{Hz}$ & 091 & ,128 & $+4 \pm 9$ & $+50 \pm 51$ & $-25 \pm 11 \#$ \\
\hline Index of B-rhythm, \% & ,103 &,- 025 & $+12,5 \pm 6,0^{\#}$ & $+2,8 \pm 5,8$ & $-2,0 \pm 4,0$ \\
\hline Testosterone, nM/L & 096 &,- 103 & $2,9 \pm 1,4^{\#}$ & $-6,8 \pm 4,7$ & $-3,4 \pm 2,3$ \\
\hline Baevskiy's Stress Index, ln units &,- 394 & 047 & $-0,57 \pm 0,14^{\#}$ & $+0,04 \pm 0,04$ & $+0,61 \pm 0,08^{\#}$ \\
\hline Entropy of SPD in P4 &,- 111 &,- 019 & $-0,06 \pm 0,02^{\#}$ & $-0,05 \pm 0,05$ & $+0,01 \pm 0,02$ \\
\hline Popovych's Strain Index, units &,- 081 & ,135 & $-0,29 \pm 0,14^{\#}$ & $+0,24 \pm 0,23$ & $-0,03 \pm 0,05$ \\
\hline T5-6 SPD, \% &,- 077 & 034 & $-8,4 \pm 3,0^{\#}$ & $-1,4 \pm 4,6$ & $-0,5 \pm 3,7$ \\
\hline CD8+CD3+ Tc-Lymphocytes, \% &,- 114 &,- 059 & $-1,1 \pm 0,8$ & $-1,7 \pm 1,9$ & $+1,3 \pm 0,6^{\#}$ \\
\hline Asymmetry of B-rhythm, \% &,- 076 & 035 & $-7,4 \pm 4,9$ & $-0,3 \pm 5,6$ & $+0,4 \pm 2,2$ \\
\hline F7- $\theta$ SPD,$\mu V^{2} / \mathrm{Hz}$ &,- 078 &,- 030 & $-0,5 \pm 3,0$ & $-1,1 \pm 4,7$ & $+6,6 \pm 3,5$ \\
\hline F4- $\delta$ SPD, $\mu V^{2} / \mathrm{Hz}$ &,- 064 & 012 & $-65 \pm 65$ & $+65 \pm 78$ & $+152 \pm 143$ \\
\hline Active T-Lymphocytes, $\%$ &,- 052 &, 053 & $-1,2 \pm 0,7$ & $+0,4 \pm 0,6$ & $-0,2 \pm 0,7$ \\
\hline Fp1- $\delta$ SPD, \% &,- 003 & 137 & $+1,7 \pm 5,4$ & $+26,6 \pm 16,3$ & $+4,1 \pm 4,1$ \\
\hline IgA, g/L &, 005 &, 103 & $-0,03 \pm 0,14$ & $+0,40 \pm 0,41$ & $-0,01 \pm 0,23$ \\
\hline $\begin{array}{l}\text { Popovych's Adaptation Index, } \\
\text { points }\end{array}$ &, 051 & 084 & $+0,16 \pm 0,10$ & $+0,49 \pm 0,31$ & $-0,01 \pm 0,15$ \\
\hline Frequency of $\alpha$-rhythm, $\mathrm{Hz}$ &,- 011 & ,074 & $-0,17 \pm 0,23$ & $+0,50 \pm 0,34$ & $-0,24 \pm 0,06^{\#}$ \\
\hline
\end{tabular}




\begin{tabular}{|c|c|c|c|c|c|}
\hline F8-6 SPD, $\mu V^{2} / \mathrm{Hz}$ &, 013 &, 033 & $-11 \pm 18$ & $+3 \pm 6$ & $-15 \pm 12$ \\
\hline Fp1-a SPD, \% &, 055 &,- 131 & $+2,0 \pm 2,8$ & $-13 \pm 9$ & $-3,6 \pm 2,5$ \\
\hline Entropy of SPD in O1 &,- 035 &,- 109 & $+0,03 \pm 0,04$ & $-0,08 \pm 0,06$ & $+0,05 \pm 0,02^{\#}$ \\
\hline IgM, g/L &,- 020 &,- 108 & $+0,10 \pm 0,11$ & $-0,25 \pm 0,24$ & $+0,12 \pm 0,08$ \\
\hline Entropy of SPD in F4 &, 033 &,- 054 & $+0,03 \pm 0,03$ & $-0,04 \pm 0,04$ & $0,00 \pm 0,03$ \\
\hline \multicolumn{3}{|c|}{$\begin{array}{l}\text { Chi Square Tests with Successive Roots } \\
\text { Removed }\end{array}$} & \multicolumn{3}{|c|}{ Squared Mahalanobis Distances } \\
\hline \begin{tabular}{r|} 
Eigenvalues \\
Canonical $\mathrm{R}$ \\
Wilks' $\Lambda$ \\
\end{tabular} & $\begin{array}{l}8,31 \\
, 945 \\
, 018 \\
\end{array}$ & $\begin{array}{l}5,02 \\
, 913 \\
, 166 \\
\end{array}$ & \multicolumn{3}{|c|}{$\begin{array}{l}\text { V-N: } 60\left(\mathrm{~F}=4,4 ; p<10^{-3}\right) \\
\text { S-N: } 61\left(\mathrm{~F}=4,6 ; p<10^{-4}\right) \\
\text { V-S: } 38\left(\mathrm{~F}=7,8 ; p<10^{-6}\right)\end{array}$} \\
\hline$x^{2}$ & 153 & 66 & & & \\
\hline Degree Freedom & 54 & 26 & & & \\
\hline $\mathrm{p}$-level & $<10^{-6}$ & $<10^{-5}$ & & & \\
\hline
\end{tabular}

The localization of the points of a neutral cluster along the axis of the first root in its quasi-zero zone reflects, as a rule, quasi-zero changes of the mentioned parameters, but with many exceptions. On the contrary, along the axis of the second radical, the neutral cluster is distanced very clearly from the other two, which, in turn, are not delimited completely. This reflects the increase in the values of the parameters positively correlated with the second root and the decrease in the values of negatively correlated with its parameters in comparison with quasi-zero changes in both the vagotonic and sympatotonic vegetotropic reactions.

By means of calculating the classifying functions for their coefficients and constants (Table 11) as well as individual values of postprandial changes, the selected discriminant variables allow to identify retrospectively the variant of the vegetotropic reaction to the stimulus without mistakes.

Table 11

Coefficients and Constants for Classification Functions

\begin{tabular}{|c|c|c|c|}
\hline \multirow{2}{*}{ Variables currently in the model } & Vagotonic & Neutral & Sympathotonic \\
\hline & $p=, 39$ & $p=, 11$ & $p=, 50$ \\
\hline Baevskiy's Stress Index, ln units & $-5,701$ & 9,881 & 9,438 \\
\hline Triangulary Index, units & 1,357 &,- 422 &,- 696 \\
\hline $\mathrm{T} 5-\alpha \mathrm{SPD}, \mu \mathrm{V}^{2} / \mathrm{Hz}$ &,- 027 & ,066 & ,006 \\
\hline T5-8 SPD, \% &,- 207 & 273 & 212 \\
\hline F7- $\theta \mathrm{SPD}, \mu \mathrm{V}^{2} / \mathrm{Hz}$ &,- 205 & ,153 & ,214 \\
\hline Fp1- $\delta$ SPD, \% &, 041 &,- 078 &, 130 \\
\hline Frequency of a-rhythm, $\mathrm{Hz}$ &,- 734 & 7,082 &,- 118 \\
\hline Stub Neutrophils, \% & ,934 & $-2,896$ & ,373 \\
\hline HF SP, $\mathrm{msec}^{2}$ & 0029 &,- 0018 &,- 0087 \\
\hline Popovych's Adaptation Index, points & $-6,171$ & 32,98 & 7,903 \\
\hline Asymmetry of B-rhythm, \% &,- 096 & ,232 & 034 \\
\hline CD8+CD3+ T cytolytic Lymphocytes, $\%$ &,- 430 &,- 212 & ,695 \\
\hline Entropy of SPD in O1 & 3,971 & $-36,92$ & 16,96 \\
\hline Popovych's Strain Index, units & $-3,862$ & 16,99 & $-2,927$ \\
\hline Testosterone, nM/L &,- 359 & 1,368 & 439 \\
\hline F8-6 SPD, $\mu V^{2} / \mathrm{Hz}$ &, 027 &,- 025 &,- 031 \\
\hline
\end{tabular}



https://

\begin{tabular}{|l|c|c|c|}
\hline F4- $\delta$ SPD, $\mu \mathrm{V}^{2} / \mathrm{Hz}$ &, 0023 &, 0011 &,- 0008 \\
\hline F7- $\mathrm{SPD}, \%$ &,- 109 & 1,266 &,- 278 \\
\hline Fp1-a SPD, \% &, 171 & $-1,124$ &, 298 \\
\hline T5-a SPD, \% &,- 045 &,- 453 &, 078 \\
\hline LF SP, msec ${ }^{2}$ &,- 0019 &, 0116 &,- 0004 \\
\hline IgA, g/L &, 086 & 10,32 & $-1,224$ \\
\hline IgM, g/L &, 518 & 4,780 & $-3,507$ \\
\hline Entropy of SPD in F4 & 18,89 & 44,80 & $-9,331$ \\
\hline "Active" T-Lymphocytes, \% &,- 390 & $-1,661$ &, 133 \\
\hline Entropy of SPD in P4 & $-26,87$ & $-20,54$ & 2,054 \\
\hline Index of B-rhythm, \% &, 0358 &, 0799 &,- 0365 \\
\hline Constants & $-6,758$ & $-21,97$ & $-6,16$ \\
\hline
\end{tabular}

Fig. 2 illustrates the previously mentioned complete absence of differences between the three stimuli within each variant of the vegetotropic response to them. If the similarity of reactions to bioactive waters Naftussya and phytoadaptogen was expected due to the presence of polycyclic hydrocarbons in both stimuli [7, 11, 12, 22, 23], then the vegetotropic activity of ordinary waters caught me by surprise.

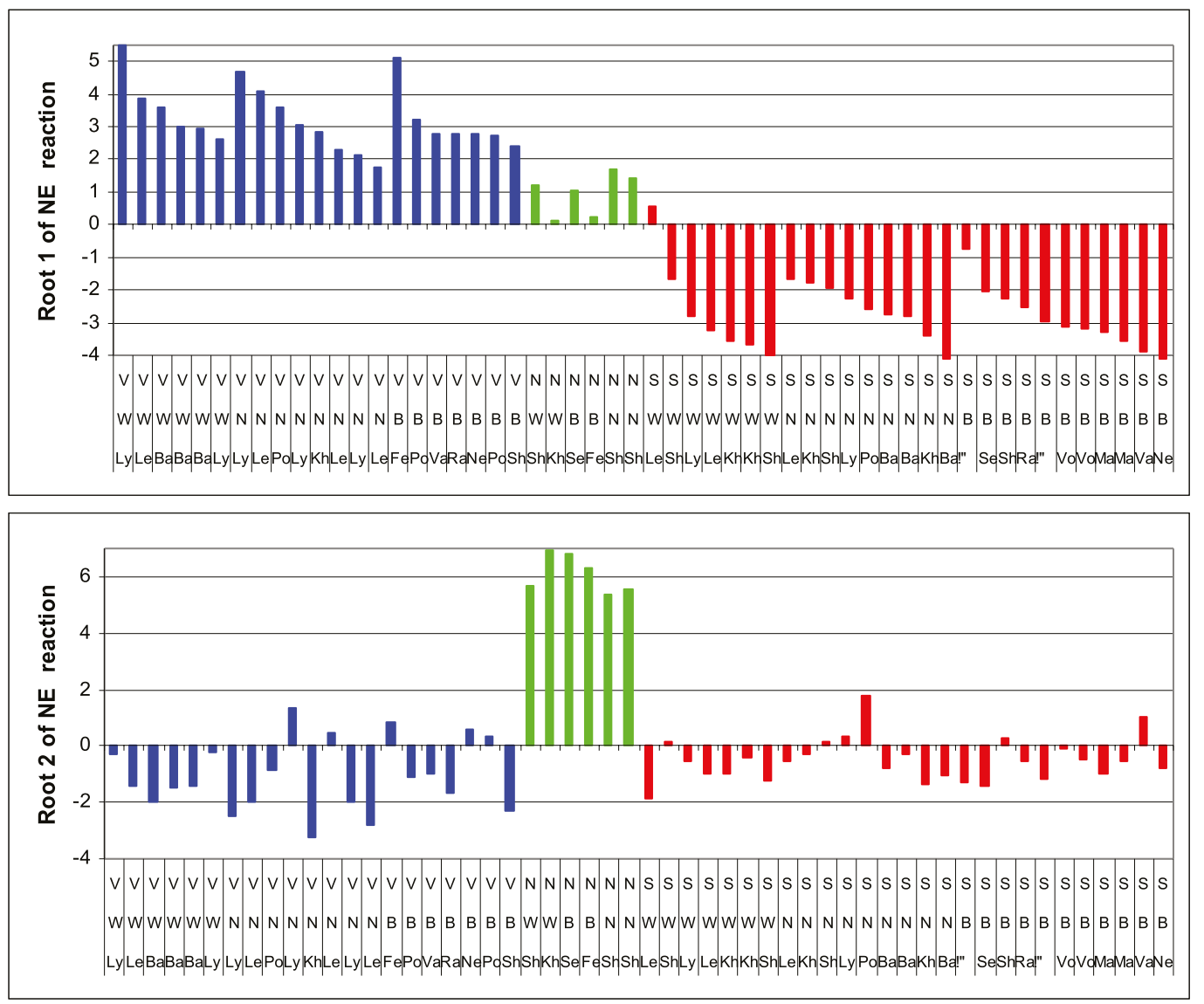

Fig. 2. Individual values of first and second roots of vagotonic $(\mathrm{V})$, neutral $(\mathrm{N})$ and sympathotonic (S) vegetotropic reactions to the intake of ordinary waters $(\mathrm{W})$, bioactive waters Naftussya $(\mathrm{N})$ and balsam (B). The volunteers' codes are indicated 
The hypothesis should be accepted that the vector of the vegetotropic reaction as well as its absence, is due not to the properties of the stimulus, but to the state of the autonomic reactivity of a person and not at all, especially at the time of using the fluid.

The procedure of the discriminatory analysis of registered initial parameters revealed their constellation (2 of HRV, 21 of EEG and 8 of Immunity as well as Trait anxiety) which allows to predict each of the three variants of the vegetotropic reaction accurately (Table 12).

Table 12

Coefficients and constants for classification of functions and means of predictors of various vegetotropic reaction

\begin{tabular}{|c|c|c|c|c|c|c|c|}
\hline \multirow{2}{*}{$\begin{array}{l}\text { Variables-predictors } \\
\text { currentlyin the model }\end{array}$} & \multicolumn{3}{|c|}{$\begin{array}{l}\text { Coefficients for } \\
\text { reactions }\end{array}$} & \multicolumn{3}{|c|}{ Means of predictors of reaction } & \multirow{2}{*}{$\begin{array}{l}\text { Norm } \\
\text { levels }\end{array}$} \\
\hline & V & $\mathbf{N}$ & $\mathbf{S}$ & Sympath & Vagotonic & Neutral & \\
\hline Amplitude of Moda, \% & 6,54 & 6,90 & 7,11 & $43 \pm 3$ & $66 \pm 5$ & $55 \pm 6$ & $37,8 \pm 1,3$ \\
\hline C4- $\theta$ SPD, \% & $-36,15$ & $-34,69$ & $-39,25$ & $9,6 \pm 0,7$ & $12,8 \pm 1,1$ & $11,5 \pm 1,8$ & $9,4 \pm 0,3$ \\
\hline Asymmetry of B-rhythm, \% & $-11,57$ & $-11,16$ & $-12,61$ & $16 \pm 2$ & $24 \pm 59$ & $28 \pm 10$ & $20 \pm 1,5$ \\
\hline Entropy of SPD in C4 & 2476 & 2357 & 2512 & $0,84 \pm 0,02$ & $0,89 \pm 0,02$ & $0,89 \pm 0,03$ & $0,83 \pm 0,01$ \\
\hline Circulating Immune Comp, un & ,95 & ,93 & ,96 & $38 \pm 3$ & $74 \pm 24$ & $42 \pm 4$ & $54 \pm 5$ \\
\hline C4- $\delta$ SPD, \% & $-12,96$ & $-11,30$ & $-12,52$ & $20,2 \pm 2,2$ & $25,1 \pm 2,5$ & $21,4 \pm 2,3$ & $21,6 \pm 1,2$ \\
\hline "Active" T-Lymphocytes, \% & 121 & 116 & 127 & $25,6 \pm 0,6$ & $26,6 \pm 1,1$ & $26,8 \pm 1,5$ & $29,6 \pm 0,8$ \\
\hline Triangulary Index, units & 44,12 & 43,97 & 47,85 & $10,9 \pm 0,8$ & $7,4 \pm 0,7$ & $7,9 \pm 1,1$ & $11,2 \pm 0,3$ \\
\hline Stub Neutrophils, \% & 97,97 & 96,59 & 104,6 & $4,30 \pm 0,36$ & $2,86 \pm 0,37$ & $3,52 \pm 0,28$ & $3,50 \pm 0,23$ \\
\hline Asymmetry of a-rhythm, \% & 3,80 & 3,26 & 3,98 & $18,4 \pm 2,4$ & $14,7 \pm 1,1$ & $10,3 \pm 0,6$ & $17,0 \pm 1,1$ \\
\hline Index of B-rhythm, \% & $-2,89$ & $-2,35$ & $-2,97$ & $92,0 \pm 2,8$ & $76,1 \pm 6,0$ & $91,3 \pm 5,7$ & $87,9 \pm 1,8$ \\
\hline O1-a SPD, \% & 5,40 & 5,01 & 5,74 & $48 \pm 5$ & $33 \pm 5$ & $44 \pm 8$ & $48 \pm 3$ \\
\hline Killing Index of Neutroph, \% & 10,68 & 10,01 & 10,96 & $38 \pm 2$ & $32 \pm 4$ & $33 \pm 4$ & $40 \pm 2$ \\
\hline 0-Lymphocytes, \% & $-13,65$ & $-11,81$ & $-14,11$ & $16,2 \pm 1,7$ & $14,2 \pm 1,2$ & $12,9 \pm 4,0$ & $8,0 \pm 0,8$ \\
\hline Laterality Index of a-rhythm, $\%$ & $-3,20$ & $-3,51$ & $-3,66$ & $-3 \pm 4$ & $+8 \pm 4$ & $-14 \pm 3$ & $-2 \pm 2$ \\
\hline Fp1- $\delta$ SPD, \% & 29,80 & 28,33 & 31,37 & $18 \pm 3$ & $22 \pm 4$ & $11 \pm 2$ & $18,9 \pm 1,4$ \\
\hline Immunoglobulins $\mathrm{A}, \mathrm{g} / \mathrm{L}$ & $-37,90$ & $-63,88$ & $-73,40$ & $1,27 \pm 0,05$ & $1,40 \pm 0,074$ & $1,19 \pm 0,15$ & $1,90 \pm 0,06$ \\
\hline T3- $\delta$ SPD, \% & $-1,66$ & $-1,96$ & $-2,07$ & $21 \pm 3$ & $27 \pm 4$ & $18 \pm 4$ & $20,2 \pm 1,4$ \\
\hline Frequency of $\theta$-rhythm, $\mathrm{Hz}$ & 127,6 & 115,4 & 130,3 & $6,4 \pm 0,3$ & $6,6 \pm 0,2$ & $5,8 \pm 0,6$ & $6,5 \pm 0,1$ \\
\hline F8- $\delta \mathrm{SPD}, \mu \mathrm{V}^{2} / \mathrm{Hz}$ & 1,14 & 1,03 & 1,20 & $43 \pm 10$ & $82 \pm 35$ & $21 \pm 6$ & $71 \pm 14$ \\
\hline P3- $\theta$ SPD, \% & 26,86 & 24,01 & 29,41 & $9,0 \pm 0,9$ & $10,0 \pm 1,2$ & $7,3 \pm 0,9$ & $7,6 \pm 0,3$ \\
\hline Fp1- $\delta$ SPD, $\mu V^{2} / \mathrm{Hz}$ & $-2,72$ & $-2,63$ & $-2,82$ & $51 \pm 13$ & $87 \pm 40$ & $21 \pm 5$ & $63 \pm 13$ \\
\hline F4- $\delta$ SPD, $\mu V^{2} / \mathrm{Hz}$ & 22 & ,19 & ,31 & $77 \pm 14$ & $150 \pm 70$ & $55 \pm 14$ & $89 \pm 9$ \\
\hline T4- $\delta$ SPD, $\mu V^{2} / \mathrm{Hz}$ &,- 11 &,- 19 &,- 54 & $48 \pm 7$ & $80 \pm 28$ & $43 \pm 10$ & $73 \pm 12$ \\
\hline F7- $\delta$ SPD, $\mu V^{2} / \mathrm{Hz}$ &,- 02 &,- 15 &,- 15 & $40 \pm 8$ & $60 \pm 19$ & $31 \pm 8$ & $72 \pm 14$ \\
\hline T6- $\delta$ SPD, $\mu V^{2} / \mathrm{Hz}$ & $-3,06$ & $-2,71$ & $-3,08$ & $38 \pm 7$ & $59 \pm 21$ & $30 \pm 8$ & $64 \pm 11$ \\
\hline Laterality Index of $\delta$-rhythm, $\%$ & 3,13 & 2,97 & 3,32 & $-7 \pm 9$ & $0 \pm 8$ & $-16 \pm 6$ & $+1 \pm 4$ \\
\hline Trait anxiety, points & $-5,15$ & $-5,31$ & $-6,20$ & $46,9 \pm 1,2$ & $48,4 \pm 1,5$ & $45,8 \pm 3,0$ & $38,0 \pm 1,2$ \\
\hline Theophill resistance T-Lym, \% & 3,14 & 4,10 & 3,84 & $22,0 \pm 1,2$ & $21,9 \pm 0,80$ & $30,0 \pm 4,5$ & $33,2 \pm 1,2$ \\
\hline Eosinophils, \% & $-28,89$ & $-24,40$ & $-32,21$ & $3,50 \pm 0,15$ & $3,33 \pm 0,21$ & $4,83 \pm 1,14$ & $3,50 \pm 0,23$ \\
\hline Entropy of SPD in F8 & -415 & -344 & -417 & $0,78 \pm 0,03$ & $0,76 \pm 0,05$ & $0,86 \pm 0,05$ & $0,76 \pm 0,02$ \\
\hline Deviation of $\delta$-rhythm, $\mathrm{Hz}$ & 74,18 & 68,43 & 71,30 & $0,79 \pm 0,05$ & $0,76 \pm 0,07$ & $0,92 \pm 0,15$ & $0,70 \pm 0,03$ \\
\hline Constants & -3214 & -3019 & -3407 & & & & \\
\hline
\end{tabular}




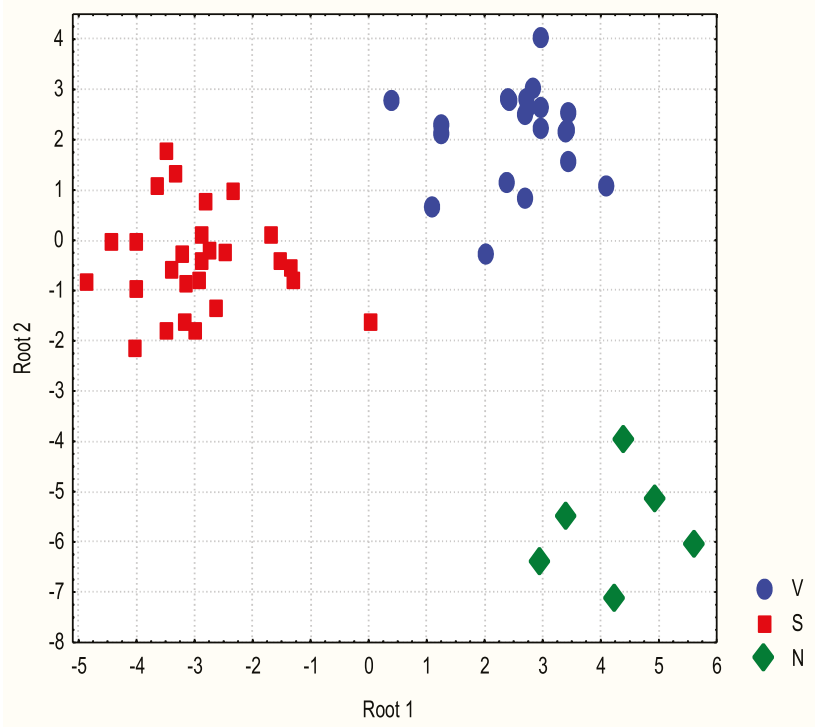

Fig. 3. Localization in a two-dimensional information space individual values of roots of initial neuroendocrine-immune parameters that determine various vegetotropic reactions

As we can see (Fig. 3) the sympathetic response to the stimulus has developed in the cases of minimum/maximum for a sample of initial values of the parameters associated with the first root. The neutral response is determined by the minimum/maximum values of the parameters associated with the second root, whereas the predictors of the vagotonic reaction are the maximal/ minimal of these parameters.

Conclusion. The vector of the vegetotropic reaction as well as its absence is due not to the properties of the stimulus, but to the state of autonomic reactivity of a person, and not at all, especially at the time of using the fluid.

Acknowledgement. I express my sincere gratitude to my colleague T.A. Korolyshyn as well as the administration of the JSC 'Truskavets'kurort' and the clinical sanatorium 'Moldova' for help in recording EEG and HRV and carrying out immuno-enzyme analysis. Special thanks to the volunteers who have became my friends.

Compliance with ethical standards. Tests of patients were conducted in accordance with the Declaration of Helsinki (1975), which was revised and complemented in 2002 and the directive of the Science Research Ethics Committee. During the tests the informed consent has been taken from all participants' parents and all necessary measures have been used for providing the anonymity of participants.

Authors state no conflict of interests.

\section{REFERENCES}

1. Baevskiy RM, Ivanov GG. Heart Rate Variability: theoretical aspects and possibilities of clinical application [in Russian]. Ultrazvukovaya i funktsionalnaya diagnostika. 2001;3:106-27. 2. Balanovs'kyi VP, Popovych IL, Karpynets' SV. About ambivalence-equilibratory character of influence of curative water Naftussya on organism of human [in Ukrainian]. Dopovidi ANU. Matematichni, pryrodnychi, tekhnicni Nauky. 1993;3:154-8. 3. Barylyak LG, Malyuchkova RV, Tolstanov OB, Tymochko OB, Hryvnak RF, Uhryn MR. Comparative estimation of informativeness of leucocytary index of adaptation by Garkavi and by Popovych. Medical Hydrology and Rehabilitation. 2013;11(1):5-20. 4. Berntson GG, Bigger JT, Eckberg DL, Grossman P, Kaufman PG, Malik M, Nagaraja HN, Porges SW, Saul JP, Stone PH, Van der Molen MW. Heart Rate Variability: Origines, methods, and interpretive caveats. Psychophysiology. 1997;34:623-48. 
5. Bilas VR, Popovych IL. Role of microflora and organic substances of water Naftussya in its modulating influence on neuroendocrine-immune complex and metabolism [in Ukrainian]. Medical Hydrology and Rehabilitation. 2009;7(1): 68-102. 6. Chebanenko OI, Chebanenko LO, Popovych IL. Variety Balneoeffects of Factors Spa Truskavets' and their Forecast [in Ukrainian]. Kyiv: UNESCO-SOCIO: 2012. 496p. 7. Dats'ko OR, Bubnyak AB, Ivassivka SV. The organic part in mineral water Naftussya. Development of knowledges about its composition and origination [in Ukrainian]. Medical Hydrology and Rehabilitation. 2008;6(1):168-174. 8. Douglas SD, Quie PG. Investigation of Phagocytes in Disease. Churchil. 1981. 110p. 9. Gumega MD, Levyts'kyi AB, Popovych IL. Balneogastroenterology [in Ukrainian]. Kyiv: UNESCO-SOCIO. 2011. $243 \mathrm{p} .10$. Heart Rate Variability. Standards of Measurement, Physiological Interpretation, and Clinical Use. Task Force of ESC and NASPE. Circulation. 1996;93(5):1043-1065. 11. Ivassivka SV. Biological Active Substances of Water Naftussya, their Genesis and Mechanisms of Physiological Effects [in Ukrainian]. Kyiv: Naukova dumka. 1997. 110p. 12. Ivassivka SV, Bubnyak AB, Kovbasnyuk MM, Popovych IL. Genesis and role of phenols in waters from Naftussya layer [in Ukrainian]. In: Problems of pathology in experiment and clinic. Scientific works of Drohobych Medical Institute. Vol. XV. Drohobych. 1994:6-11. 13. Klecka WR. Discriminant Analysis [trans. from English in Russian] (Seventh Printing, 1986). In: Factor, Discriminant and Cluster Analysis. Moskwa: Finansy i Statistika. 1989: 78-138. 14. Kozyavkina NV, Gozhenko AI, Barylyak LG, Korolyshyn TA, Popovych IL. Variety immediate thyrotropic effects of bioactive water Naftussya, their neuroendocrine-immune accompaniment and possibility of forecast [in Ukrainian]. Medical Hydrology and Rehabilitation. 2013;11(4):27-54. 15. Kozyavkina OV, Kozyavkina NV, Gozhenko OA, Gozhenko AI, Barylyak LG, Popovych IL. Bioactive Water Naftussya and NeuroEndocrine-Immune Complex [in Ukrainian]. Kyiv: UNESCO-SOCIO. 2015. 349p. 16. Kozyavkina $O V$, Popovych IL, Zukow W. Immediate vegetotropic effects of bioactive water Naftussya and their neuro-endocrine-immune accompaniment in healthy men. Journal of Health Sciences. 2013;3(5):391-408. 17. Lapovets' LY, Lutsyk BD. Handbook of Laboratory Immunology [in Ukrainian]. L'viv: 2002. 173p. 18. Newberg AB, Alavi A, Baime M, Pourdehnad M, Santanna $J$, d'Aquili $E$. The measurement of regional cerebral blood flow during the complex cognitive task of meditation: a preliminary SPECT study. Psychiatry Research: Neuroimaging Section. 2001;106:113-22. 19. Panasyuk YM, Levkut LH, Popovych IL, Alekseyev OI, Kovbasnyuk MM, Balanovskyi VP. Experimental study of adaptogenic properties of 'Crimean' balm [in Ukrainian]. Fiziol Zh. 1994;40(3-4):25-30. 20. Petsyukh SV, Petsyukh MS, Kovbasnyuk MM, Barylyak LG, Zukow W. Relationships between Popovych's Adaptation Index and parameters of ongoiging HRV and EEG in patients with chronic pyelonephrite and cholecystite in remission. Journal of Education, Health and Sport. 2016;6(2):99-110. 21. Popovych IL. Functional relationships between parameters of neuro-endocrine-immune complex at male rats [in Ukrainian]. Achivements of Clinical and Experimental Medicine. 2008;2(9):80-7. 22. Popovych IL. Stresslimiting Adaptogene Mechanism of Biological and Curative Activity of Water Naftussya [in Ukrainian]. Kyiv: Computer press: 2011. 300p. 23. Popovych IL, Ivassivka SV. Role of organic substances of water Naftussya in its physiological activity [in Ukrainian]. Medical Hydrology and Rehabilitation. 2009;7(2):6-26. 24. Popovych IL, Kozyavkina $O V$. Term vegetotropic effects bioactive water Naftussya and their neuro-endocrine-immune accompaniment in apparently healthy men [in Ukrainian]. Medical Hydrology and Rehabilitation. 2012;10(3):32-65. 25. Popovych IL, Kozyavkina OV, Kozyavkina NV, Korolyshyn TA, Lukovych YS, Barylyak LG. Correlation between Indices of the Heart Rate Variability and Parameters of Ongoing EEG in Patients Suffering from Chronic Renal Pathology. Neurophysiology. 2014;46(2):139-48. 26. Popovych IL, Lukovych YS, Korolyshyn TA, Barylyak $L G$, Kovalska LB, Zukow W. Relationship between the parameters heart rate variability and background EEG activity in healthy men. Journal of Health Sciences. 2013;3(4):217-40. 27. Popovych IL, Ruzhylo SV, Ivassivka SV, Aksentiychuk BI. (editors). Balneocardioangiology [in Ukrainian]. Kyiv: Computerpress. 2005. 229p. 28. Popovych IL, Vis'tak HI, Gumega MD, Ruzhylo $S V$. Vegetotropic Effects of Bioactive Water Naftussya and their Endocrine-Immune, Metabolic and Hemodynamic Accompaniments [in Ukrainian]. Kyiv: UNESCO-SOCIO; 2014. 163p. 29. Practical psychodiagnostics. Methods and tests [in Russian]. Samara: Publing House Bakhrakh; 1998:59-64. 30. Shannon CE. Works on the theory of informatics and cybernetics [transl. from English to Russian]. Moskwa: Inostrannaya literatura; 1963. 329p. 31. Vovchyna YV, Lukovych YR, Matiyishyn GY, Hryvnak RF, Tymochko OO, Andrusiv OV, Burkovs'ka MM. Hemodynamic variants term effects of bioactive water Naftussya on blood pressure, their electrolyte accompaniment and the ability to forecast [in Ukrainian]. Medical Hydrology and Rehabilitation. 2013;11(3):50-9. 


\title{
RESEARCH ARTICLES
}

\section{Immediate responses of the autonomic nervous system to the balneofactors, their neuro-endocrine-immune accompaniments and predictors}

\author{
I.L. POPOVYCH \\ Bohomolets' Institute of Physiology of National Academy of Sciences, Kyiv, Ukraine \\ email: i.popovych@biph.kiev.ua
}

Introduction. In previous studies it has been shown that in response to the intake of Bioactive Water Naftussya (BAWN) the activity of the autonomic nervous system changes and the vector of the reaction is ambiguous. However, the issue of the specificity of immediate effects of BAWN on the nervous as well as endocrine and immune systems is still relevant. This is the objective of this study.

Material and Methods. The object of the observation was 15 volunteers-men (aged $26 \div 60$, $\mathrm{M} \pm \mathrm{SD}$ : $44 \pm 12$ years) without any clinical diagnose but with a moderate dysfunction of neuroendocrine-immune complex (dysadaptation). At the beginning volunteers filled in a questionnaire with the purpose of estimationing the level of the trait anxiety. Then HRV and EEG were recorded. The content of principal adaptation hormones such as Cortisol, Testosterone and Triiodothyronine as well as parameters of Immunity were determined in blood. After registration 5 volunteers of the basic level consumed some days $200 \mathrm{~mL}$ of Control Water (distillated, filtered, well), Water Naftussya from layers Truskavets' and Skhidnyts'a while 10 volunteers consumed $5 \mathrm{~mL}$ of Phytocomposition 'Balm Cryms'kyi' (it is identified as an adaptogen) soluted in $195 \mathrm{~mL}$ of daily Water. all tests were repeated in an hour and a half.

Results. It has been confirmed that the polyvariant nature of the vegetotropic reactions to the balneofactors, namely Bayevskiy's Stress Index has increased in half of the observations, while in $39 \%$ it has decreased and only in $11 \%$ has not changed significantly. No differences were found between the effects of all applied stimuli. The method of discriminant analysis revealed 27 parameter change which are characteristic of vegetotropic reactions (Baevskiy's Stress Index, 3 parameters of HRV, 15 of EEG and 7 of Immunity as well as Testosterone). It has been revealed that initial parameters ( 2 of HRV, 21 of EEG and 8 of Immunity as well as trait anxiety) allowed to predict each of the three variants of the vegetotropic reaction accurately.

Conclusion. The vector of the vegetotropic reaction as well as its absence, is not due to the properties of the stimulus, the time of the use of the balneofactor, but to the state of autonomic reactivity of a person.

Key words: balneofactors, neuroendocrine-immune complex, immediate reactions. 